\title{
Detection and Counting of On-Tree Citrus Fruit for Crop Yield Estimation
}

\author{
Zeeshan Malik, Sheikh Ziauddin, Ahmad R. Shahid, and Asad Safi \\ Department of Computer Science, \\ COMSATS Institute of Information Technology, \\ Park Road, Islamabad
}

\begin{abstract}
In this paper, we present a technique to estimate citrus fruit yield from the tree images. Manually counting the fruit for yield estimation for marketing and other managerial tasks is time consuming and requires human resources, which do not always come cheap. Different approaches have been used for the said purpose, yet separation of fruit from its background poses challenges, and renders the exercise inaccurate. In this paper, we use k-means segmentation for recognition of fruit, which segments the image accurately thus enabling more accurate yield estimation. We created a dataset containing 83 tree images with 4001 citrus fruits from three different fields. We are able to detect the on-tree fruits with an accuracy of $91.3 \%$. In addition, we find a strong correlation between the manual and the automated fruit count by getting coefficients of determination $R^{2}$ up to 0.99 .
\end{abstract}

Keywords-Precision agriculture; yield estimation; $k$-means segmentation; leaf occlusion; illumination; morphology

\section{INTRODUCTION}

In citrus groves, yield estimation is typically carried out a few weeks earlier to fruitage to estimate the resource requirement. The manual process of crop assessment is occasionally done by hand pawns. Preferably, yield would be estimated at numerous periods during crop development but it requires enormous labor cost and time. Precise, low-cost yield estimation is important for cultivators, especially if it can be done timely in the growing season. As orange juice needs to be processed within 48 hours of harvesting, orange juice manufacturers need suppliers to provide accurate yield estimates to guarantee that their juice plants can run at full capacity given the time constraints. Additionally, more accurate yield estimates will allow farmers to plan more precisely for their harvesting labor and other logistical needs. Image processing can help in improving the decision making process for irrigation, fruit sorting and yield estimation [1]. Detection of fruit is important as the subsequent fruit counting depends on accurate detection.

Citrus fruit have different properties that can be used for detection purposes. The most natural property to be used for such purpose is the color. In the past, color has been extensively used [2], [3], [4], [5], [6], [7], [8], [9]. Color on is own may not provide enough information as it may change depending upon how ripe the fruit is. For instance, unripe oranges may be greenish, while over-ripe oranges may be brownish. That poses a challenge when detection is based on filtering of orange color only.

Lighting may pose another challenge as the oranges may appear differently, under varying lighting conditions, such as bright sunlight, cloudy, and evening. Figure 1 shows a tree image under two varying light conditions. The image on the left is captured on a cloudy day and the one on the right is captured under direct sunlight. Under cloudy conditions, the images are more consistent in terms of brightness and intensity changes. While in the latter case, things may look different if they are exposed to direct sunlight as opposed to when they are under a shadow.

Another problem is that of occlusion. Occlusion may be caused either by leaves or by the neighboring oranges, as can be seen from Figure 2. In the first case, a single orange may be counted as more than one, as leaves may affect the shape of the orange. In the later case, may fruits may appear as one larger fruit with irregular shape. In the first case, a need arises for a way to count two or more smaller citrus blobs as one, while in the later case we need to break down a larger blob into smaller units, where each unit is counted as a separate fruit.

\section{RELATED WORK}

Despite the above-mentioned challenges involved, image processing has been used with good results for automated fruit counting and yield estimation. In the past, a variety of techniques have been used across diverse fruits. Stajnko et al. [10] used a combination of normalized chromaticity coordinates (RGB) and thermal images to estimate on-tree count of apple fruit. In order to separate pixels more precisely, they also used the normalized difference index (NDI). Using their technique, they were able to achieve correlation coefficient $R^{2}$ of 0.83 to 0.88 at different times during the vegetation period. They observed that the values of $R^{2}$ were improved during the fruit ripening period. In a subsequent work [11], they used surface texture and color features to segment apples from the background. HSI color space was used for segmentation using a threshold which was empirically selected. Red color intensity $(3 * R-G-B)$, contrast, uniformity and entropy were the main features used. They achieved a detection accuracy of $89 \%$ with a false detection of $2.2 \%$.

Zhou et al. [12] also used color features for on-tree apple counting. Fifty tree images were captured at two different times during the season. The images were captured under normal daylight conditions. For the images captured earlier in the season, they used color RGB difference $(R-B$ and $G-R)$ while two different color models were used for the images captured during ripening season. They achieved $R^{2}$ scores of 0.8 and 0.85 for the two seasons, respectively. Regunathan and Lee [13] presented a technique for citrus detection and 

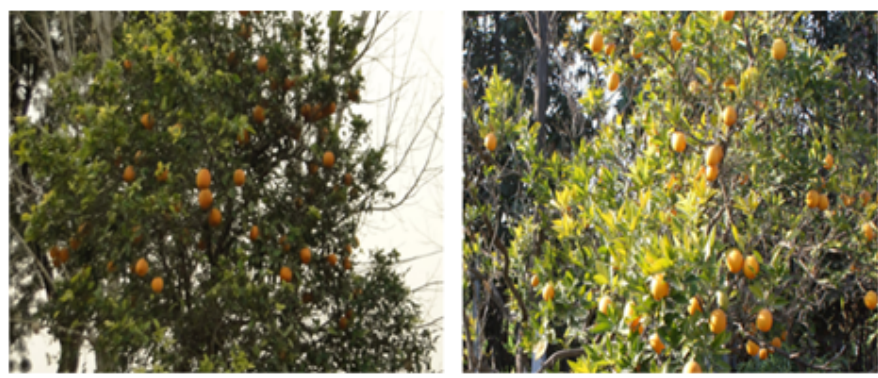

Fig. 1: Citrus tree images captured under two different sunlight conditions.

size finding. Their technique relies on an untrasonic sensor to determine the distance between the camera and the fruit. They used hue and saturation features in combination with three different classifiers namely Beyesian, neural network and Fischer's linear discriminant to segment and differentiate fruit from the background.

Hannan et al. [14] used red chromaticity coefficient for image enhancement which helped in better segmentation in variable light orange tree images. They used shape analysis technique to detect overlapped oranges achieving a detection rate of $90 \%$ with $4 \%$ false detection. Yet another citrus fruit counting scheme was presented by Chinchuluun et al. [15]. They used Bayesian classification in addition to morphological operations and watershed segmentation for citrus segmentation and detection. They reported a correlation coefficient value of 0.89 between the manual and the automated fruit count. Billingsley [16] presented a machine vision system for counting Macadamia nuts on a harvester roller. RGB color features were used to separate the nuts and the leaves from the roller. To segment the nuts from the leaves, they used a modified version of circular Hough transform. Cakir et al. [17] used color histograms and shape analysis to detect and count orange fruit on tree images. They were able to achieve a detection accuracy of $76.5 \%$.

Annamalai and Lee [18] developed an algorithm for citrus counting. They used hue and saturation color planes and utilized histogram information to segment the fruit from the background and the leaves. Erosion and dilation operations were used to remove noise. They found $R^{2}$ of 0.76 between the manual count and the count obtained using their algorithm.

In this paper, we present a technique for automated yield estimation using k-means segmentation. The proposed technique is able to detect and count citrus fruit by catering for changes in color both due to lighting conditions as well as the state of ripeness of the fruit. Our system also takes care of the cases where the fruit is occluded either by the leaves or by other fruit. The experimental evaluation shows the accuracy and robustness of the proposed scheme as described in the subsequent sections.

The rest of this paper is organized as follows: The proposed scheme is presented in Section 2. The results of our experiments are described in Section 3 while Section 4 concludes this paper.
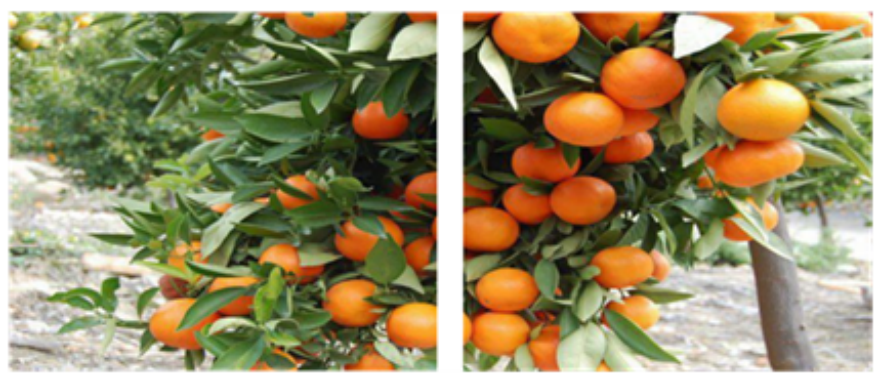

Fig. 2: Orange fruit occluded by leaves (left). Orange fruit overlapping each other (right).

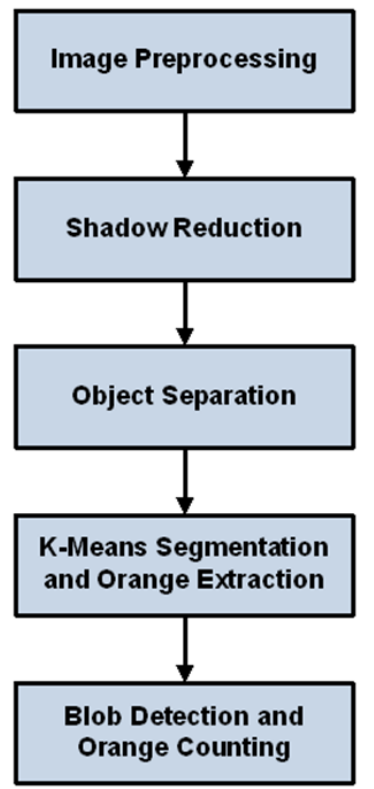

Fig. 3: An overview of the proposed technique.

\section{Proposed Technique}

The technique we proposed here uses k-means segmentation algorithm on orange tree images. First, a few preprocessing steps are performed including noise reduction and image enhancement. Next, we minimize shadow effects from the image. Then, we extract oranges using blob detection and size calculation which is then followed by the final yield estimation. Figure 3 gives an overview of the proposed technique by showing its different steps in a sequential order. The details of these steps are given in the following sub-sections.

1) Image Preprocessing: In general, images contain a lot of redundant information not needed for the application in hand. The image may contain noise which makes the edge detection and the segmentation tasks prone to errors. Therefore, it is often necessary to perform certain type of noise reduction and image enhancement before any meaningful processing of the images. In this paper, we use the Perona-Malik model for image enhancement and noise reduction [19]. It smoothes the image without effecting significant features of the image such as lines, edges or other important details that are necessary for analysis and interpretation of the images. In this model, image is smoothed using the following mathematical relation. 

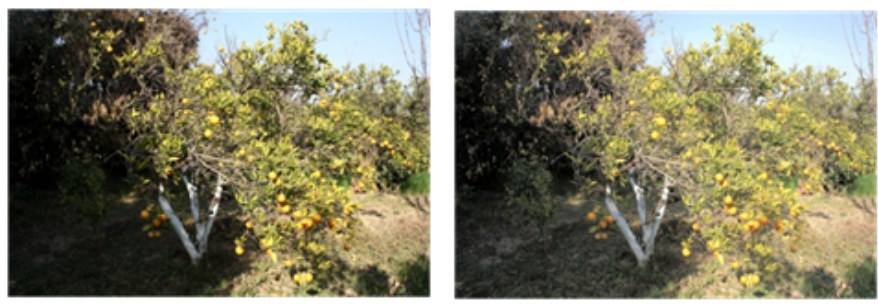

Fig. 4: Shadow reduction step. The image on left is before the shadow reduction while that on right is the same image after shadow reduction step is applied.

$$
\frac{\partial I}{\partial t}=\operatorname{div}(c(x, y, t) \nabla I)=c(x, y, t) \Delta I+\nabla c . \Delta I
$$

where div denotes the divergence operator, while $\nabla$ and $\Delta$ represents gradient and Laplacian, respectively. The diffusivity function in Perona-Malik model is given by the following equation.

$$
c(x, y, t)=g(\|\nabla I(x, y, t)\|)
$$

where $g$ is a nonnegative, monotonically decreasing function with $g(0)=1$.

\section{A. Shadow Reduction}

Lighting conditions play a very important role in the performance of many computer vision algorithms. In particular, shadows in an image can cause problems in recognition, segmentation and tracking algorithms to produce desirable outcomes. Distinct objects can be combined through shadows and they can obscure object recognition systems. When dealing with outdoor images, shadow minimization is an important task. In order to minimize shadow, we adjust the luminosity of the image. First, we convert the RGB image to $L * a * b$ image, where $L$ is the luminosity layer, while $a$ and $b$ represents color-opponent dimensions. Next, we increase the luminosity of the image which results in reduced shadow effect in the image. Finally, the image is converted to RGB color space after replacing the luminosity layer with the processed data. Figure 4 shows an image after shadow reduction step is applied.

\section{B. Object Separation}

One of the main challenges in orange counting is that of overlapped oranges. Due to overlapping, multiple fruits may be counted as a single fruit which negatively impacts the fruit counts and the yield estimates. In order to overcome this challenge and to separate the overlapping fruit, we convolve the image with a variance mask. After the convolution, each pixel in the output RGB image contains the neighbor variance of the R, G and B channels, respectively [20]. The image is then transformed to gray scale by taking the mean of the three color channels. Finally, a threshold is applied on the image. These steps not only separate overlapping fruit, they also help in reducing undesired edges such as those within the foliage or the grass. Figure 5 shows an image after object separation step is applied.
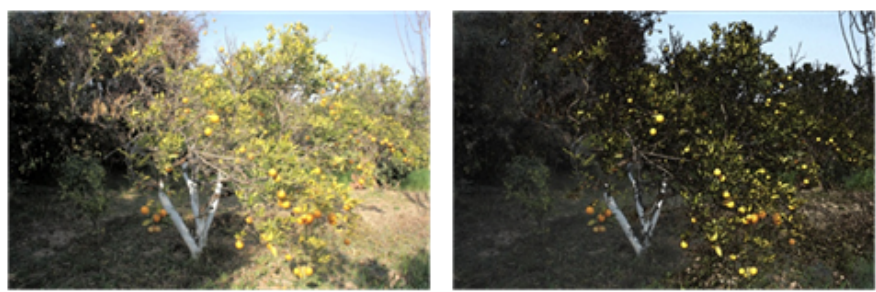

Fig. 5: Object separation step. The image on left is before the object separation while that on right is the same image after object separation step is applied.

\section{K-Means Segmentation and Orange Extraction}

Image Segmentation is the most important part of the whole process of yield estimation. We use k-means clustering algorithm for orange segmentation. K-means clustering is an unsupervised classification technique which deals with finding a pattern in a collection of unlabelled data [21]. The k-means algorithm aims at minimizing a squared error function by iteratively reorganizing the clusters. The iterations continue until the cluster means do not move further than a certain cut-off value.

$\mathrm{K}$-means algorithm is popular due to its simplicity and relatively low computational complexity. It is suitable in our scenario as the number of clusters $(\mathrm{K})$ is easy to choose. An image of an orange tree generally consists of regions representing the oranges, leaves, branches and sky. Therefore, we select $K$ to be 4 corresponding to these 4 regions. After the clustering, we apply thresholding to extract the oranges from the tree images. Each object in the image is segmented using a particular RGB value.

In many cases, the fruit is visually fragmented because of the obstructions caused by the leaves. This makes the counting error prone as one fruit may be counted as two or more fruits. In order to remove the smaller fragments of these fruits, we converted the image into binary and applied the erosion operation. The erosion of a binary image $A$ by the structuring element $B$ is defined by [22]:

$$
A \ominus B=\left\{z \mid(B)_{z} \subseteq A\right\}
$$

i.e., the output of erosion functions contains all pixels $z$ in $A$ such that $B$ is in $A$ when origin of $B=z$.

Figure 6 shows two images. On the left, there is input image while the output of k-means segmentation is shown on the right.

\section{Blob Detection and Orange Counting}

The final step of the proposed orange counting technique is to detect monolithic fruit regions, which are also known as blobs or objects. After the erosion operation, we find the connected components in the binary image using 8-connectivity. Each connected component corresponds to one orange. We count the number of connected components which gives us the number of oranges in a particular tree image. 

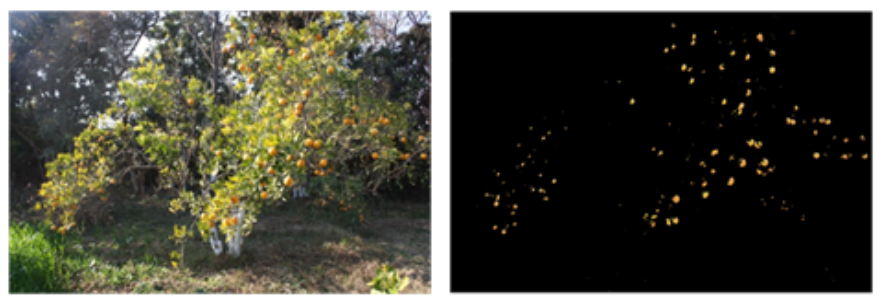

Fig. 6: K-means segmenation step. The image on left is the input image while that on right is the output after $\mathrm{k}$-means segmentation is applied.

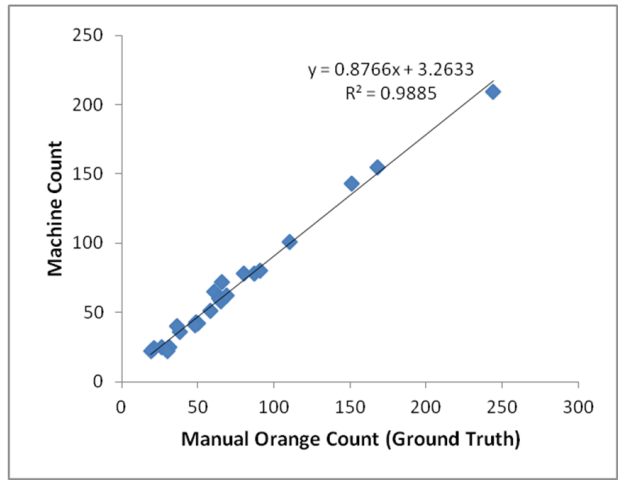

Fig. 7: Comparison of citrus fruit count per tree counted manually against that calculated using the proposed technique for Khanpur dataset.

\section{EXPERIMENTAL EVALUATION}

\section{A. Dataset}

We have collected a total of 83 tree images taken from Khapur and National Agricultural Research Council (NARC) fields. The images were taken at three times with an average gap of around one month. In the rest of this paper, we will call these three datasets Khanpur, NARC 1 and NARC 2, respectively. Khanpur dataset contains 23 tree images while NARC 1 and NARC 2 contain 16 and 44 tree images, respectively. The images contain variable illumination including shadows, bright sunlight and dusk. We prepared a ground truth by manually counting and marking all the orange fruit in all the input images.

\section{B. Results}

We performed experiments by getting automated citrus counting using the proposed scheme and comparing the results with the ground truth. The experimental results show a correct detection rate of $91.3 \%$, as shown in Table I. In addition to finding detection rate, we used linear regression to model the relationship between the automated results and the ground truth. Figures 7, 8, and 9, show the result of plotting manual and automated orange counts for Khanpur, NARC 1 and NARC 2 datasets, respectively. The figures also show the regression equations as well as the coefficient of determination $R^{2}$. As you can see, neither the accuracy in Table I, nor the coefficients of determination in Figures 7, 8, 9 vary significantly among different datasets. As the datasets were created at different

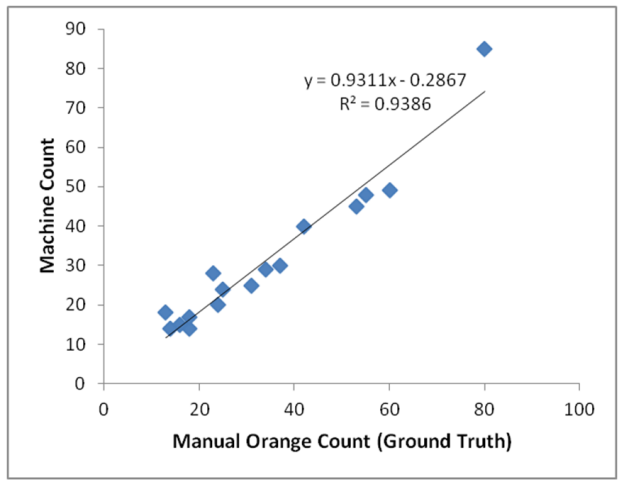

Fig. 8: Comparison of citrus fruit count per tree counted manually against that calculated using the proposed technique for NARC 1 dataset.

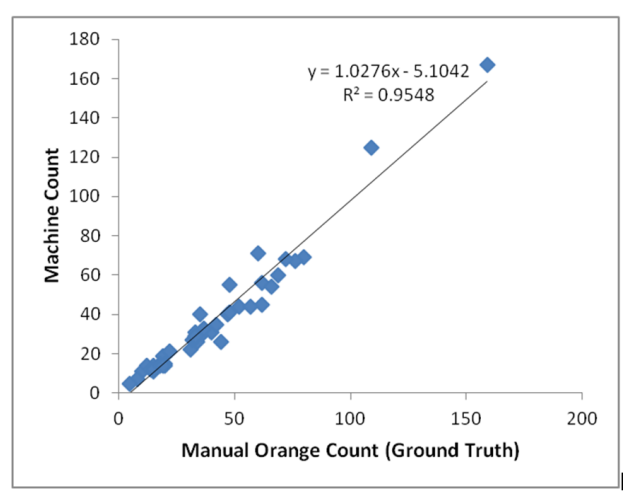

Fig. 9: Comparison of citrus fruit count per tree counted manually against that calculated using the proposed technique for NARC 2 dataset.

times with varying lighting conditions, the consistency in results shows the robustness of the proposed technique.

In Table II, we compare our results with the past work. Some of the past work has presented results in terms of detection accuracy while some has presented it in terms of coefficient of determination $\left(R^{2}\right)$. Table II shows that our technique is capable of detecting and counting the fruits with more accuracy as compared to others.

\section{CONCLUSiON}

In this paper, we have presented a technique for the segmentation, detection and yield measurement of citrus fruit. The proposed approach gives very good results under varying lighting conditions, occlusion of leaves and overlapping of

TABLE I: Orange detection and counting results of the proposed scheme.

\begin{tabular}{ccccc}
\hline Dataset & No of Images & No of Fruits & Machine Count & Accuracy (\%) \\
\hline Khanpur & 23 & 1662 & 1532 & 92.2 \\
NARC 1 & 16 & 543 & 501 & 92.3 \\
NARC 2 & 44 & 1796 & 1621 & 90.3 \\
Overall & $\mathbf{8 3}$ & $\mathbf{4 0 0 1}$ & $\mathbf{3 6 5 4}$ & $\mathbf{9 1 . 3 0 \%}$ \\
\hline
\end{tabular}


TABLE II: Comparison with the previous work.

\begin{tabular}{ccc}
\hline Technique & Accuracy (\%) & $\boldsymbol{R}^{\mathbf{2}}$ Value \\
\hline Cakir et al. [17] & 76.5 & - \\
Hannan et al. [14] & 90 & - \\
Chinchuluunet al. [15] & - & 0.89 \\
Annamalai and Lee [18] & - & 0.76 \\
Proposed Technique & 91.3 & 0.94 to 0.99
\end{tabular}

fruits on the images taken from varying distances from the orange trees. Our experiments on three different datasets showed an accuracy of $91.3 \%$ with $R^{2}$ values of up to 0.99 . In the future, we aim to collect larger datasets for further experiments. In addition, instead of taking images manually from the citrus trees, we plan to use a camera-mounted robot for image acquisition.

\section{REFERENCES}

[1] C. Pohl and J. Van Genderen, "Review article multisensor image fusion in remote sensing: concepts, methods and applications," International journal of remote sensing, vol. 19, no. 5, pp. 823-854, 1998.

[2] D. Bulanon, T. Kataoka, S. Zhang, Y. Ota, and T. Hiroma, "Optimal thresholding for the automatic recognition of apple fruits," ASAE, no. 01-3033, 2001

[3] T. Burks, "A real-time machine vision algorithm for robotic citrus harvesting," 2007.

[4] M. W. Hannan and T. F. Burks, "Current developments in automated citrus harvesting," in ASABE Annual International Meeting, 2004.

[5] R. Harrell, D. Slaughter, and P. Adsit, "A fruit-tracking system for robotic harvesting," Machine Vision and Applications, vol. 2, no. 2, pp. 69-80, 1989.

[6] R. Harrell, P. Adsit, T. Pool, R. Hoffman et al., "The florida robotic grove-lab." Transactions of the ASAE, vol. 33, no. 2, pp. 391-399, 1990.

[7] A. Jimenez, R. Ceres, J. Pons et al., "A survey of computer vision methods for locating fruit on trees," Transactions of the ASAE-American Society of Agricultural Engineers, vol. 43, no. 6, pp. 1911-1920, 2000.

[8] F. Pla, F. Juste, and F. Ferri, "Feature extraction of spherical objects in image analysis: an application to robotic citrus harvesting," Computers and Electronics in Agriculture, vol. 8, no. 1, pp. 57-72, 1993.

[9] E. Tutle, "Image controlled robotics in agricultural environments," 1984.

[10] D. Stajnko, M. Lakota, and M. Hočevar, "Estimation of number and diameter of apple fruits in an orchard during the growing season by thermal imaging," Computers and Electronics in Agriculture, vol. 42, no. 1, pp. 31-42, 2004.

[11] D. Stajnko, J. Rakun, M. Blanke et al., "Modelling apple fruit yield using image analysis for fruit colour, shape and texture." European journal of horticultural science, vol. 74, no. 6, pp. 260-267, 2009.

[12] R. Zhou, L. Damerow, Y. Sun, and M. M. Blanke, "Using colour features of cv. 'gala' apple fruits in an orchard in image processing to predict yield," Precision Agriculture, vol. 13, no. 5, pp. 568-580, 2012.

[13] M. Regunathan and W. S. Lee, "Citrus fruit identification and size determination using machine vision and ultrasonic sensors," in ASABE Annual International Meeting, 2005.

[14] M. Hannan, T. Burks, and D. M. Bulanon, "A machine vision algorithm combining adaptive segmentation and shape analysis for orange fruit detection," Agricultural Engineering International: CIGR Journal, 2010.

[15] R. Chinchuluun, W. Lee, R. Ehsani et al., "Machine vision system for determining citrus count and size on a canopy shake and catch harvester," Applied Engineering in Agriculture, vol. 25, no. 4, pp. 451458, 2009.

[16] J. Billingsley, "More machine vision applications in the NCEA," in Mechatronics and Machine Vision in Practice. Springer, 2008, pp. 333-343.
[17] Y. Cakir, M. Kirci, E. O. Gunes, and B. B. Ustundag, "Detection of oranges in outdoor conditions," in Agro-Geoinformatics (AgroGeoinformatics), 2013 Second International Conference on. IEEE, 2013, pp. 500-503.

[18] P. Annamalai and W. Lee, "Citrus yield mapping system using machine vision," in ASAE Annual International Meeting, 2003.

[19] P. Perona and J. Malik, "Scale-space and edge detection using anisotropic diffusion," Pattern Analysis and Machine Intelligence, IEEE Transactions on, vol. 12, no. 7, pp. 629-639, 1990.

[20] A. B. Payne, K. B. Walsh, P. Subedi, and D. Jarvis, "Estimation of mango crop yield using image analysis-segmentation method," Computers and Electronics in Agriculture, vol. 91, pp. 57-64, 2013.

[21] D. Fradkin and I. Muchnik, "A study of k-means clustering for improving classification accuracy of multi-class SVM," Technical Report. Rutgers University, New Brunswick, New Jersey 08854, Tech. Rep., 2004.

[22] R. C. Gonzalez and R. E. Woods, Digital Image Processing. Prentice Hall, 2007. 